\title{
Problèmes expérimentaux de la méthode potentiométrique avec l'électrolyte liquide lors de l'étude des alliages métalliques et semi-conducteurs
}

\author{
V. Vassiliev \\ Laboratoire de Thermodynamique Chimique, Faculté de Chimie, Université \\ Lomonossov, Moscou 119992, Russie
}

\begin{abstract}
This paper reviews some methodological problems and experimental sources of errors which are encountered when using electrochemical cells with liquid electrolytes to determine thermodynamic properties of alloys. Indications to get reliable results are given.
\end{abstract}

\section{INTRODUCTION}

La méthode de mesure des forces électromotrices (FEM) de piles à électrolyte liquide est un instrument puissant pour étudier les propriétés thermodynamiques des systèmes métalliques et semi-conducteurs. Elle permet de déterminer un ensemble de valeurs du potentiel chimique de l'un des composants dans les différentes phases du système étudié. Le résultat final de ce travail consiste à optimiser numériquement le système du point de vue thermodynamique.

Nous envisageons dans cette communication certains problèmes méthodologiques et les sources d'erreurs qui apparaissent lors des mesures de FEM des piles électrochimiques et les moyens de les surmonter.

\subsection{Principe de la méthode}

On réalise des mesures de potentiel aux bornes de cellules électrochimiques, avec électrolyte liquide, du type :

(-) $\mathrm{A} \mid \mathrm{A}^{(\mathrm{n}+)}$ dans 1' électrolyte $\mid \mathrm{A}_{\mathbf{x}} \mathrm{B}_{(1-\mathrm{x})}(+)$

$\mathrm{x}$ représente la fraction molaire du composant $\mathrm{A}$ dans 1'alliage liquide ou solide $\mathrm{A}_{\mathrm{x}} \mathrm{B}_{(1-\mathrm{x})}$.

Le composant $A$ (en général un métal pur) constitue 1'électrode négative, 1'alliage $A_{x} B_{(1-x)}$ dans lequel le composant $B$ est plus noble que $A$, constitue 1'electrode positive. Le potentiel chimique du métal $\mathrm{A}, \mu_{A}^{(A)}$, est toujours supérieur à son potentiel chimique $\mu_{A}^{\left(A_{4} B_{(1-x)}\right)}$ dans 1'alliage $\mathrm{A}_{\mathrm{x}} \mathrm{B}_{(1-\mathrm{x})}$ soit $\quad\left(\mu_{A}^{(A)}\right)>\mu_{A}^{\left(A_{u} B_{(1-x)}\right)}$

$\Delta \mu_{\mathrm{A}}=\mathrm{RT} \operatorname{Ln}\left(\left(a_{A}^{\left(A_{x} B_{(1-x)}\right)}\right) / a_{A}^{(A)}\right)=\operatorname{RT} \operatorname{Ln} \mathrm{a}^{\prime \prime} / \mathrm{a}^{\prime}$

Si $a_{A}^{(A)}=1$, c'est à dire si l'on effectue les mesures de forces électromotrices entre le métal pur A et 1'alliage, il vient :

$\Delta \mu_{\mathrm{A}}=\mathrm{RT} \operatorname{Ln}\left(a_{A}^{\left(A_{x} B_{(1-x)}\right)}\right)$

$\Delta \mu_{\mathrm{A}}$ est la variation du potentiel chimique du composant $\mathrm{A}$ entre le metal pur et 1'alliage $\mathrm{A}_{\mathrm{x}} \mathrm{B}_{1-\mathrm{x}}$ dans les conditions de référence. La mesure de $\mathrm{E}$ en fonction de la temperature mène aux fonctions thermodynamiques partielles. 


\subsection{Différents types des piles électrochimiques}

Il existe de nombreux exemples de construction de piles électrochimiques proposés dans la littérature. Nous avons réalisé, essayé et utilisé sur différents alliages trois types de piles en 'Pyrex', dont la conception nous est propre. L'un des trois convient bien jusqu'à $200^{\circ} \mathrm{C}$ [1-3] et les deux autres fonctionnent aux températures plus élevées, jusqu'au ramollissement du 'Pyrex' $\left(560-600^{\circ} \mathrm{C}\right)$ [4-9]. On peut également exploiter les mêmes types de constructions, en verre réfractaire ou en quartz, jusqu'à $1000^{\circ} \mathrm{C}[10]$.

\subsection{Préparation de l'électrolyte}

Il est nécessaire d'apporter une attention particulière à la préparation des sels de l'électrolyte. Ils doivent être desséchés très soigneusement. Ceci concerne surtout les compositions à base d'halogénures de $\mathrm{Li}, \mathrm{Ca}, \mathrm{Zn}$ et $\mathrm{Al}$. Ces halogénures sont extrêmement hygroscopiques et leur fusion, sans déshydratation quasi-totale au préalable, aboutit à la formation d'oxyhalogènures dont il faut éviter la présence. L'électrolyte utilisé doit être parfaitement transparent à l'état liquide et ne présenter aucun trouble ou hétérogénéité. Les lingots d'électrolyte de réserve se conservent dans des ampoules en Pyrex scellées. Lors de l'introduction de l'électrolyte dans une pile, le contact avec l'air doit être minimal (pas plus de quelques secondes).

L'étude de certains systèmes, comme les chalcogénures de zinc, cadmium, mercure, thallium, bismuth, etc. (binaires ou multinaires), peut être réalisée dans une pile du type «basse température " avec un électrolyte formé d'une solution de $\mathrm{CaCl}_{2}$ dans le glycérol, entre 40 et $180^{\circ} \mathrm{C}$. Il est préférable d'utiliser les électrodes sous forme de pastilles de poudre frittée [1]. Parfois l'utilisation d' échantillons sous forme massive altère les résultats de mesure de la FEM, surtout si ces mesures sont faites à température beaucoup plus basse que celles auxquelles se forment les phases étudiées [11].

\section{LES PROBLEMES METHODOLOGIQUES LES PLUS SOUVENT RENCONTRES}

1) Réactions d'échanges entre électrodes provoquées par une faible différence des potentiels d'électrodes [12] des composants de l'alliage dans l'électrolyte donné.

2) Réactions spontanées dues à une grande différence de potentiel chimique entre électrodes de mesure et de référence.

3) Présence simultanée de plusieurs degrés d'oxydation des ions impliqués dans la réaction d'oxydo-réduction générant la FEM de la pile.

\subsection{Réactions d'échange}

L'interaction entre les électrodes par l'intermédiaire de l'électrolyte est un des problèmes les plus importants. Dans l'électrolyte, A et $\mathrm{B}$ sont caractérisés par des potentiels différents vis à vis d'une électrode de référence [12]. Il faut choisir un électrolyte qui entraîne une différence des potentiels de $\mathrm{A}$ et $\mathrm{B}$ aussi grande que possible. Plus cette différence est grande, plus la réaction d'échange est petite entre le composant B et 1'ion An+ dans 1'electrolyte fondu.

$$
(1 / n B) B\left(\text { dans } A_{x} B_{(1-x)}\right)+(1 / n A) A^{n+} \rightarrow(1 / n A) A+(1 / n B) B^{n+}
$$

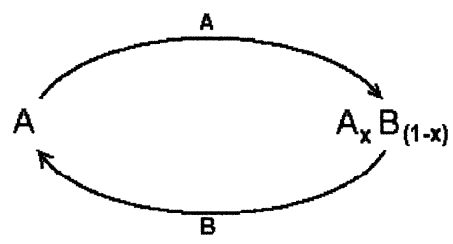


Les renseignements à propos d'une possible réaction d'échange entre électrodes peuvent être trouvés à partir des potentiels d'électrode dans les différents halogènures fondus [12].

Ces séries de potentiels caractérisent 1'activite chimique des métaux les uns par rapport aux autres, pour le système étudié et dans un électrolyte donne. Plus un métal est électronégatif plus il est chimiquement actif : en particulier dans une série de potentiel chaque métal remplace dans $1^{\prime}$ électrolytes tous les métaux de potentiel inférieur et, à son tour, il est remplacé, dans le même électrolyte, par les métaux de potentiel supérieur. Plus un métal est électropositif, plus il est chimiquement actif. En particulier, dans un électrolyte donné, le métal de potentiel le plus négatif remplace tous ceux de potentiels plus élevés.

$\mathrm{Si}$ l'on étude les systèmes binaires $\mathrm{Zn}-\mathrm{Sb}$ ou In-Sb par la méthode potentiométrique on ne remarque pas de problème de réaction d'échange. Donc, on peut inférer que si la différence des potentielles électrochimiques atteint $0.4 \mathrm{~V}$, les réactions d'échange sont négligeables. Le problème apparaît pour les systèmes $\mathrm{Zn}$-In et In-Sn, lesquels présentent une différence de potentiels électrochimiques de $0.19 \mathrm{~V}$. Lors de l'étude des piles de type :

(-) $\mathrm{Zn} \mid \mathrm{Zn}^{2+}$ dans l'électrolyte $\mid \mathrm{Zn}_{\mathrm{x}} \mathrm{In}_{(1-\mathrm{x})}(+)$

$(-) \operatorname{In} \mid \operatorname{In}^{+}$dans l'électrolyte $\mid \operatorname{In}_{\mathrm{x}} \operatorname{Sn}_{(1-\mathrm{x})}(+)$

les réactions d'échange se déroulent facilement dès que la concentration du deuxième élément atteint 90 at\%. La chute continue de la FEM des piles réalisées avec des alliages où $\mathrm{x}_{\leq} 0.1$ est observée si la durée de l'expérience dépasse quelques semaines $[8,13]$. La vitesse de la réaction d'échange augmente avec l'accroissement de la température surtout dans les systèmes liquides.

La vitesse de la réaction d'échange dépend :

1) de la différence des potentiels électrochimiques des éléments,

2) de la différence de composition des alliages,

3) de la présence d'ions métalliques de charges différentes $\left(\mathrm{A}^{(\mathrm{n}+)}\right.$ et $\left.\mathrm{A}^{(\mathrm{m}+)}\right)$ dans l'électrolyte

4) de la température de l'expérience,

\subsection{Influence d'un troisième composant sur la réaction d'échange}

Les réactions d'échange se produisent non seulement entre un métal $A$ pur et son alliage $A_{x} B_{(1-x)}$ mais aussi entre des alliages de différentes compositions, si les activités $\mathrm{a}_{\mathrm{A}} \mathrm{y}$ sont très différentes. $\mathrm{Ce}$ phénomène est très prononcé dans le système ternaire liquide $\mathrm{In}-\mathrm{Sn}-\mathrm{Sb}$ [9].

Le tableau 1 illustre que l'attraction entre atomes In et $\mathrm{Sb}$ est plus grande que celle entre In et $\mathrm{Sn}$ ou bien entre $\mathrm{Sn}$ et $\mathrm{Sb}$. En conséquence, les atomes $\mathrm{Sn}$ de l'alliage ternaire sont très libres et les réactions d'échange entre des électrodes d'alliages de compositions différentes, avec de l'indium, se produisent facilement.

Tableau 1.

\begin{tabular}{|c|c|c|}
\hline Système & $\begin{array}{c}\Delta \mathrm{G}_{\text {mix }}(900 \mathrm{~K}) \\
\mathrm{J} / \text { mole-atome }\end{array}$ & Référence \\
\hline $\mathrm{Sn}-\mathrm{Sb}$ & -2340 & {$[7]$} \\
\hline $\mathrm{In}-\mathrm{Sn}$ & -4310 & {$[8]$} \\
\hline $\mathrm{In}-\mathrm{Sb}$ & -9070 & {$[14]$} \\
\hline
\end{tabular}

La cinétique des réactions d'échange entre les électrodes: In (pur), $\operatorname{In}_{0.05} \mathrm{Sn}_{0.45} \mathrm{Sb}_{0.5}$ et $\mathrm{In}_{0.5} \mathrm{Sn}_{0.25} \mathrm{Sb}_{0.25}$ a été examinéedans une pile avec un électrolyte de sels fondus [9].

Deux réactions d'échange principales se déroulent en même temps entre ces électrodes :

a)

$$
\begin{aligned}
& \text { In(référence) }{ }^{\mathrm{In} \rightarrow} \underset{\mathrm{a}_{\text {In }}=1}{\leftarrow \text { Sn }}\left(\mathrm{In}_{0.05} \mathrm{Sn}_{0.45} \mathrm{Sb}_{0.5} ; \mathrm{In}_{0.5} \mathrm{Sn}_{0.25} \mathrm{Sb}_{0.25}\right) \\
& \mathrm{a}_{\text {In }}^{\prime}<1
\end{aligned}
$$

b) $\quad \mathrm{In}_{0.5} \mathrm{Sn}_{0.25} \mathrm{Sb}_{0.25}{ }^{\mathrm{In} \rightarrow} \leftarrow \mathrm{Sn} \mathrm{In}_{0.05} \mathrm{Sn}_{0.45} \mathrm{Sb}_{0.5}$

$$
a_{\text {In }}^{\prime \prime}<a_{\text {In }}^{\prime}
$$


Les réactions du type a) diminuent la FEM des alliages, tandis que celles du type b) augmentent la FEM pour des alliages riches en indium et diminuent la FEM pour des alliage pauvres en indium. La vitesse de la réaction a) est supérieure à celle de b).

\subsection{Choix des différents types d'halogénures comme électrolytes.}

Il est possible d'utiliser les différents halogénures en qualité d'électrolytes : chlorures, bromures et iodures. Tous ces sels sont hygroscopiques et leur traitement par l'halogénure d'hydrogène correspondant $(\mathrm{HCl}, \mathrm{HBr}, \mathrm{HI})$ est nécessaire pour éviter les traces d'eau. Si un halogénure ne convient pas à cause d'une réaction d'échange on le remplace par un autre.

Le choix de l'électrolyte est déterminé par sa température de fusion et par le besoin de minimiser la réaction d'echange.

L'étude du système Sn-Sb n'est pas possible dans les chlorures fondus (la difference des potentiels d'électrodes est de $0.055 \mathrm{~V}$ ). Le remplassement des chlorures par les iodures augmente significativement cette difference jusqu'à $0.168 \mathrm{~V}$ (voir le Tabl.3) et diminue les réactions d'échanges :

$\mathrm{SnI}_{2}+\mathrm{Pb} \rightarrow \mathrm{PbI}_{2}+\mathrm{Sn}$

$\mathrm{PbI}_{2}+\mathrm{Sn} \rightarrow \mathrm{SnI}_{2}+\mathrm{Pb}$,

Bien qu'il soit impossible d'éliminer complètement leur influence, surtout pour les alliages riches en étain, la chaîne électrochimique du type : (-) $\mathrm{Pb}\left|\mathrm{KI}+\mathrm{LiI}+\mathrm{PbI}_{2}\right| \mathrm{Pb}_{\mathrm{x}} \mathrm{Sn}_{1-\mathrm{x}}(+)$ peut être étudiée.

\subsection{Problèmes relatifs à la valence de 1 'ion $\mathrm{A}^{\mathrm{n}+}$}

Pour obtenir de bons résultats expérimentaux, il est nécessaire dans la cellule du type I que la charge de l'ion responsable de la FEM soit rigoureusement connue. Pourtant, la différence d'activités du métal $A$ pur et dans 1'alliage $A_{x} B_{(1-x)}$ peut avoir pour conséquence d'entrainer des charges différentes de l'ion $A^{n+}$ au voisinage des électrodes $A$ et $A_{x} B_{(1-x)}$. Dans ce cas, même en circuit ouvert, un transfert spontané du composant $A$ vers 1'alliage $A_{x} B_{(1-x)}$ est possible et une dérive constante de la FEM se produit en fonction du temps. Ceci concerne les métaux dont les ions peuvent avoir dans l'electrolyte des charges de valeurs différentes $m>n$.

Sur la cathode: $\quad(m-n) A+n A^{m+} \rightarrow m^{n+}$

Sur 1'anode: $\quad \mathrm{mA}^{\mathrm{n}+} \rightarrow(\mathrm{m}-\mathrm{n}) \mathrm{A}+\mathrm{nA}^{\mathrm{m}+}$

Il y a transfert, en circuit ouvert, du composant $A$ du métal pur vers son alliage $A_{x} B_{(1-x)}$. Près de la cathode, la fraction des ions de charge $(\mathrm{n}+)$ sera plus grande, plus petite aux environs de l'alliage $A_{x} B_{(1-x)}$. Il y a dissolution du métal pur et dépôt sur la surface de 1'alliage $A_{x} B_{(1-x)}$. Le transfert s'effectue d'autant plus vite que la température $T$ et la concentration des ions $\mathrm{A}^{\mathrm{m}+}$ sont plus élevées et la distance entre les électrodes plus petite. Le métal A est donc transféré à travers 1'electrolyte par les ions de plus basse charge.

Si 1'activité du composant $\mathrm{A}$, dans la cellule du type (I), est réduite, il est possible de déterminer les propriétés thermodynamique même en cas de transfert spontané de A. Il est alors nécessaire de prendre pour électrode de référence un alliage binaire $A_{x} C_{(1-x)}$ dont les propriétés thermodynamiques sont bien connues, au lieu du métal pur $\mathrm{A}$.

On a alors

$\Delta \mu_{\mathrm{A}}=\operatorname{RT} \operatorname{Ln}\left(\mathrm{a}^{\prime \prime} / \mathrm{a}^{\prime}\right)$

1'activite a' est alors inférieure à 1.La différence de potentiel chimique de $\mathrm{A}$ entre les électrodes et donc la force électromotrice seront plus petites.

Ce problème est rencontré lors de l'étude du système $\operatorname{Ln}-\mathrm{Te}(\mathrm{Ln}=$ lanthanoïde $)$ avec la cellule :

(-) $\operatorname{Ln} \mid \operatorname{Ln}^{(3+)}$ dans 1'electrolyte $\mid \operatorname{Ln}_{\mathrm{x}} \mathrm{Te}_{(1-\mathrm{x})}(+)$ 


$$
\begin{aligned}
& \Delta \mu_{A}=R T \cdot \operatorname{Ln}\left(a_{A}^{A_{x} B_{(1-x)}} / a_{A}^{A_{x} C_{(1-x)}}\right) \\
& a_{A}^{A_{x} C_{(1-x)}}<a_{A}^{(A)}=1
\end{aligned}
$$

Les alliages riches en métal $\mathrm{B}$ (c'est à dire en tellure) génèrent une force électromotrice $\mathrm{E}$ de $1.5 \mathrm{~V}$. La substitution du lanthanide par un de ses alliages avec de 1'indium, alliage étudié préalablement, permet les mesures avec la cellule électrochimique suivante :

$(-) \operatorname{LnIn}_{2,5}+\operatorname{In} \mid \mathrm{La}^{(3+)}$ dans 1'electrolyte $\mid \operatorname{Ln}_{\mathrm{x}} \mathrm{Te}_{(1-\mathrm{x})}(+)$

La force électromotrice de la pile est alors deux fois plus petite et le transfert spontané devient négligeable.

\section{Références}

1. V.P. Vassiliev, A.V. Nikoliskaja, Ja.I. Gerassimov, A.F. Kuznestov, Izv. AN SSSR, Neorg. Material. (rus.), 4 (7), 1040 (1968).

2. V.P. Vassiliev, A.V. Nikoliskaja, Ja.I. Gerassimov, Dokl. AN SSSR.(rus.) 188 (6), 1318 (1969).

3. V.P. Vassiliev, A.V. Nikoliskaja, Ja.I. Gerassimov, Dokl.AN SSSR.(rus.) 199 (5), 1094 (1971).

4. V.P. Vassiliev, V.I.Goriatcheva, Ja.I.Gerassimov, Vestnik Moskovskogo Universiteta (rus), serie 2, chimie, 21 (4), 339 (1980)

5. V. Vassiliev, M. Bykov, M. Gambino, J.P. Bros, Metallkunde Z., 84, 461 (1993).

6. V. Vassiliev, M. Azzaoui, J. Hertz, Z. Metallkunde, 86, 545 (1995).

7. V. Vassiliev, M. Lelaurain, J. Hertz, Alloys Comp. J., 247, 223 (1997).

8. V. Vassiliev, Y. Feutelais, M. Sghaier, B. Legendre, Thermochim. Acta., 315, 129 (1998).

9. V. Vassiliev, Feutelais Y., M. Sghaier, B. Legendre, J. Alloys Comp., 314, 197 (2001).

10. V.Vassiliev, G.F. Voronin, G. Borzone, M. Mathon, M. Gambino, J.P. Bros, Alloys Comp. J., 269, 123 (1998).

11. Terpilovski J., Zaleska E., Gawel W., Roczniki Chemii, Ann.Soc.Chim.Pol.,37, 193 (1963).

12. J. Hladik (Ed.) Phisics of Electrolytes. Thermodynamics and Electrode Processing in Solid State Electrolytes, Vol.2, Academic Press, London, 1972.

13. Z. Mozer, Metallurg.Transaction, 2, 2175 (1971).

14. R.Hultgren, P.D.Desai, D.t.Hawkins, M.Gleiser, K.K.Kelley, Properties of Binary Alloys, ASM, Metals Park, Ohio 44073 (1973). 